\title{
Conditional and Unconditional Deterministic Lower Bounds on the MSE of the Non-Uniform Linear Co-centered Orthogonal Loop and Dipole Array
}

\author{
Tao BAO and Mohammed Nabil EL KORSO
}

\begin{abstract}
The co-centered orthogonal loop and dipole (COLD) array exhibits some interesting properties, which makes it ubiquitous in the context of polarized source localization. In the literature, one can find a plethora of estimation schemes adapted to the COLD array. Nevertheless, their ultimate performance in terms of the so-called threshold region of mean square error (MSE), have not been fully investigated. In order to fill this lack, we focus, in this paper, on conditional and unconditional bounds that are tighter than the well known Cramér-Rao Bound (CRB). More precisely, we give some closed form expressions of the McAulay-Hofstetter, the Hammersley-Chapman-Robbins, the McAulay-Seidman bounds and the recent Todros-Tabrikian bound, for both the conditional and unconditional observation model. Finally, numerical examples are provided to corroborate the theoretical analysis and to reveal a number of insightful properties.
\end{abstract}

Keywords-Deterministic lower bounds, co-centered orthogonal loop and dipole array, mean square error, performance analysis, passive source localization, SNR threshold.

\section{INTRODUCTION}

Nowadays, recent source localization systems need to operate in increasingly more crowded signal environments [2]. In this context, taking into account both the polarization diversity and the spatial diversity became ubiquitous in antenna array systems and their processing as wireless communication, radar, sonar systems, etc. [2-4]. Among different types of polarization sensitive arrays, the co-centered orthogonal loop and dipole array is commonly used since it exhibits numerous interesting properties [5-7] (e.g., the constant norm of the polarization vector, the insensibility of the polarization vector w.r.t. the source localization in the plan of the antenna etc.)

In the literature, one can find a plethora of estimation schemes adapted and/or designed particularly for the COLD array [6]. Nevertheless, their ultimate performance in terms of the mean square error (MSE), especially in the non-asymptotic region (meaning for low signal-to-noise ratio (SNR) or low observations), has not been fully investigated.

We can cite $[8,9]$, in which the authors derived closed-form expression of the approximated Cramér-Rao bound (CRB) for a sufficiently large number of sensors in the context of a COLD linear and uniform array. Whereas in $[10,11]$ the authors derived, respectively, expressions of the $\mathrm{CRB}$ for a known single source and the resolution limit for two known sources, both for known polarization state parameters.

Manuscript received March 8, 2021; revised May 5, 2021.

Tao BAO is also with the Department of Electronics Engineering, Northwestern Polytechnical University, Xi'an 710129, China. Mohammed Nabil EL KORSO is with Université Paris-Ouest Nanterre La Défense, 50 rue de Sèvres, 92410 Ville d'Avray, France (email: m.elkorso@parisnanterre.fr). This paper has been partially presented in [1].

Digital Object Identifier (DOI): 10.53907/enpesj.v1i1.11
Nevertheless, to the best of our knowledge, no results concerning the breakdown prediction for the COLD linear array (possible non-uniform) can be found in the literature. To fill this lack, we focus, in this paper, on lower bounds that are tighter than the CRB. More precisely, we give some closed form expressions of the McAulay-Hofstetter (MCB), the Hammersley-ChapmanRobbins (HCRB), the McAulay-Seidman (MSB) bounds and a recently proposed Todros-Tabrikian bound (TTB), for both the commonly assumed conditional (i.e., when the signals are assumed to be deterministic) and unconditional (i.e., when the signals are assumed to be driven by a Gaussian random process) observation models with unknown direction of arrival (DOA) and unknown polarization state parameters. Such bounds are known to be efficient to delimit and predict the optimal operating zone of estimators $[12,13]$ which is given by the threshold or breakdown point, i.e., when the estimator's MSE increases dramatically.

Such deterministic lower bounds can be derived using one of the unifications given in [12,14-16]. In this paper, we adopt the Todros and Tabrikian unification in which they propose a novel class of performance lower bounds by applying a proper integral transform [15]. Using an adequate choice of the kernel of the integral transform of the likelihood-ratio function, one obtains some well known lower bounds as the MCB, HCRB, MSB and TTB.

For the rest of this paper, the following notation will be used. A lowercase bold letter denotes a vector, and an uppercase bold letter denotes a matrix. Vectors are by default in column orientation unless specified. Upper scripts ${ }^{T}{ }^{C}$ and ${ }^{H}$ are, respectively, the transpose, the conjugate and the trans-conjugate of a matrix. The operators $\operatorname{tr}\{\},.||,.\|$.$\| and \mathcal{R}($.$) represent, the trace, the$ determinant of a matrix, the Euclidean norm and the real part, respectively. $\odot$ and $\otimes$ are the Hadamard and the Kronecker product, respectively. $\boldsymbol{I}_{L}$ denotes the $L \times L$ identity matrix. $\mathbf{1}_{L}$ is the $L \times L$ matrix filled by ones. [. $]_{i}$ and $[.]_{i, j}$ denote the $i$-th element of the vector and the $i$-th row and the $j$-th column ele- 


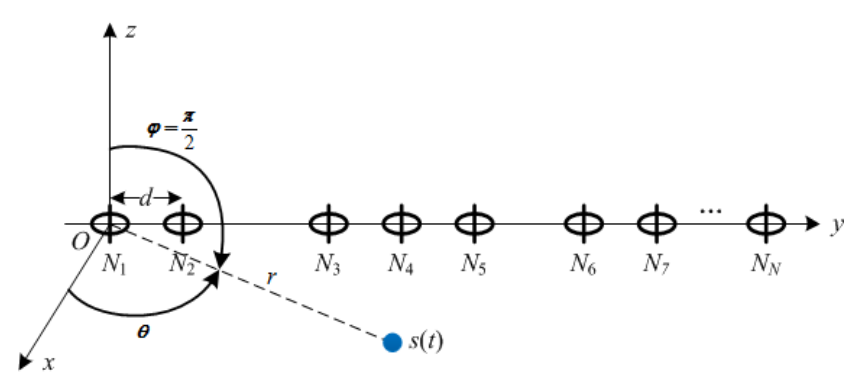

Fig. 1: A non-uniform linear COLD array representation in the presence of one far-field source

ment of the matrix. We define also the $n$-norm as $\sum \eta^{n}=\|\eta\|_{n}$. Finally, $\hat{\xi}$ is any unbiased estimate of $\boldsymbol{\xi}$.

\section{SYSTEM MODELS}

Consider a non-uniform linear array composed of $N$ COLD pairs with inter-element spacing $d_{1} . d, d_{2} . d, \ldots, d_{N} . d$ that receives a signal emitted by a single far-field and narrow-band source. Let $d_{n}$. $d$ denotes the location of the $n$-th COLD sensor, in which $d$ is unit. The array is collinear with the $y$-axis of an $(O, x y z)$ coordinate system with its origin, $O$, in the center of the first pair of sensors (i.e., $d_{1}=0$ ). For each COLD sensor, the dipole is parallel to the $z$-axis and the loop is parallel to the $x-y$ plane.

Assume a narrowband far-field source which impinges on the array from direction described by the elevation angle $\phi$ and the azimuth angle $\theta$. In this paper, we suppose that the source is contained in the $x-y$ azimuthal plane, i.e. $\phi=\frac{\pi}{2}$, as illustrated in Fig.1. For a given polarized signal, the vertical and the horizontal components of the incoming signal electric field can be specified by polarization state parameters $\rho \in\left[0, \frac{\pi}{2}\right]$ and $\psi \in[-\pi, \pi]$. Consequently, the output of the $n$-th COLD sensor can be expressed by [6]

$$
\boldsymbol{x}_{n}(t)=\left[x_{\text {loop }}, x_{\text {dipole }}\right]^{T}=s(t) \boldsymbol{u}(\psi, \rho) e^{j \omega d_{n} \cdot d}+\boldsymbol{n}_{n}(t),
$$

in which, $n=1, \ldots, N, \quad t=1, \ldots, T, x_{\text {loop }}$ and $x_{\text {dipole }}$ are the signals recorded on the small loop and the short dipole, respectively. The electrical angle $\omega=-\frac{2 \pi d}{\lambda} \sin \theta$, where $\lambda$ is the signal wavelength. $T$ is the number of snapshots and $s(t)$ is the source signal. The random process $\boldsymbol{n}_{n}(t)=\left[n_{\text {loop }}, n_{\text {dipole }}\right]^{T}$ denotes a complex Gaussian circular noise with zero mean and a known covariance matrix $\boldsymbol{\Sigma}_{\text {noise }}$. The output vector received for the $t$-th snapshot can be written as

$$
\begin{aligned}
\boldsymbol{x}(t) & =\left[\boldsymbol{x}_{1}^{T}(t), \ldots, \boldsymbol{x}_{N}^{T}(t)\right]^{T} \\
& =s(t) \boldsymbol{e}(\omega) \otimes \boldsymbol{u}(\psi, \rho)+\left[\boldsymbol{n}_{1}^{T}(t), \ldots, \boldsymbol{n}_{N}^{T}(t)\right]^{T}
\end{aligned}
$$

where the steering vector is defined by $e(\omega)=$ $\left[1, e^{j d_{2} \omega}, \ldots, e^{j d_{N} \omega}\right]^{T}$ and the $2 \times 1$ polarization state vector is given by $\boldsymbol{u}(\psi, \rho)=\left[j 2 \pi \frac{A_{s l} \cos (\rho)}{\lambda},-L_{s d} \sin (\rho) e^{j \psi}\right]^{T}$, in which $L_{s d}$ and $A_{s l}$ represent the length of the short dipole and the area of the small loop. From a modeling point of view, we can assume $L_{s d}=2 \pi \frac{A_{s l}}{\lambda}=1$.

In the following, the unknown parameter vector is given by $\boldsymbol{\xi}=[\omega, \psi, \rho]^{T}$, whereas, $\boldsymbol{\xi}_{0}, \omega_{0}, \psi_{0}$ and $\rho_{0}$ denote the real value of the candidate parameters $\boldsymbol{\xi}, \omega, \psi$ and $\rho$, respectively. The joint probability distribution function (pdf) of the full observations
$\chi=\left[\boldsymbol{x}^{T}(1), \ldots, \boldsymbol{x}^{T}(T)\right] \sim \mathcal{C N}\left(\boldsymbol{\mu}\left(\boldsymbol{\xi}_{0}\right), \boldsymbol{\Sigma}\left(\boldsymbol{\xi}_{0}\right)\right)$ for a given $\boldsymbol{\xi}_{0}$, is expressed as follow

$$
p\left(\boldsymbol{\chi} \mid \boldsymbol{\xi}_{0}\right)=\frac{1}{\left|\boldsymbol{\Sigma}\left(\boldsymbol{\xi}_{0}\right)\right| \pi^{2 N T}} e^{-\left(\boldsymbol{\chi}-\boldsymbol{\mu}\left(\boldsymbol{\xi}_{0}\right)\right)^{H} \boldsymbol{\Sigma}\left(\boldsymbol{\xi}_{0}\right)^{-1}\left(\boldsymbol{\chi}-\boldsymbol{\mu}\left(\boldsymbol{\xi}_{0}\right)\right)}
$$

Let $E\left\{\left(\hat{\boldsymbol{\xi}}_{0}-\boldsymbol{\xi}_{0}\right)\left(\hat{\boldsymbol{\xi}}_{0}-\boldsymbol{\xi}_{0}\right)^{T}\right\}$ be the covariance matrix of an estimate of $\boldsymbol{\xi}_{0}$. Let us assume that $\hat{\boldsymbol{\xi}}$ is an asymptotically unbiased estimate of the true parameter vector $\boldsymbol{\xi}_{0}$, and define the CRB for the considered model [17-19]. The covariance inequality principle states that, under quite general/weak conditions [20],

$$
\mathrm{MSE}=E\left\{\left(\left[\hat{\boldsymbol{\xi}}_{0}\right]_{i}-\left[\boldsymbol{\xi}_{0}\right]_{i}\right)^{2}\right\} \geq \operatorname{CRB}\left(\left[\boldsymbol{\xi}_{0}\right]_{i}\right), i=1,2,3
$$

where the CRB is given as the inverse of the Fisher information matrix (FIM) as $\operatorname{CRB}\left(\left[\boldsymbol{\xi}_{0}\right]_{i}\right)=\left[\mathbf{F I M}^{-1}\left(\boldsymbol{\xi}_{0}\right)\right]_{i, i}$. Since we are working with a complex circular Gaussian observation model and using the Splepian-Bang formula [21,22], the $i$-th, $k$-th element of the FIM for the unknown real parameter vector $\boldsymbol{\xi}_{0}$ can be written as

$$
\begin{aligned}
& {\left[\operatorname{FIM}\left(\boldsymbol{\xi}_{0}\right)\right]_{i, k}=} \\
& \operatorname{tr}\left\{\left.\left.\boldsymbol{\Sigma}\left(\boldsymbol{\xi}_{0}\right)^{-1} \frac{\partial \boldsymbol{\Sigma}(\boldsymbol{\xi})}{\partial[\boldsymbol{\xi}]_{i}}\right|_{\boldsymbol{\xi}=\boldsymbol{\xi}_{0}} \boldsymbol{\Sigma}\left(\boldsymbol{\xi}_{0}\right)^{-1} \frac{\partial \boldsymbol{\Sigma}(\boldsymbol{\xi})}{\partial[\boldsymbol{\xi}]_{k}}\right|_{\boldsymbol{\xi}=\boldsymbol{\xi}_{0}}\right\} \\
& +2 \mathcal{R}\left\{\left.\left.\frac{\partial \boldsymbol{\mu}^{H}(\boldsymbol{\xi})}{\partial[\boldsymbol{\xi}]_{i}}\right|_{\boldsymbol{\xi}=\boldsymbol{\xi}_{0}} \boldsymbol{\Sigma}\left(\boldsymbol{\xi}_{0}\right)^{-1} \frac{\partial \boldsymbol{\mu}(\boldsymbol{\xi})}{\partial[\boldsymbol{\xi}]_{k}}\right|_{\boldsymbol{\xi}=\boldsymbol{\xi}_{0}}\right\}
\end{aligned}
$$

There exist two different models depending on the assumptions about the signal sources, the conditional (deterministic) and unconditional (stochastic) cases.

\section{A. The Conditional Case}

The time-varying signal is modelled by $s(t)=$ $a(t) e^{j\left(2 \pi f_{0} t+\gamma(t)\right)}$, where $a(t)$ denotes the real amplitude, $\gamma(t)$ is the time-varying modulating shift phase and $f_{0}$ is the carrier frequency of the incident wave. Under this assumption, one has the following parameterized mean model given by $\chi \sim \mathcal{C N}\left(\boldsymbol{\mu}\left(\boldsymbol{\xi}_{0}\right), \boldsymbol{\Sigma}_{\text {noise }}\right)$, and in which

$$
\boldsymbol{\mu}\left(\boldsymbol{\xi}_{0}\right)=\boldsymbol{s} \otimes\left(\boldsymbol{e}\left(\omega_{0}\right) \otimes \boldsymbol{u}\left(\psi_{0}, \rho_{0}\right)\right)
$$

where $s=[s(1), \ldots, s(T)]^{T}$. Consequently, the FIM in (3) reduces to

$\left[\boldsymbol{F I M}\left(\boldsymbol{\xi}_{0}\right)\right]_{i, k}=2 \mathcal{R}\left\{\left.\left.\frac{\partial \boldsymbol{\mu}^{H}(\boldsymbol{\xi})}{\partial[\boldsymbol{\xi}]_{i}}\right|_{\boldsymbol{\xi}=\boldsymbol{\xi}_{0}} \boldsymbol{\Sigma}\left(\boldsymbol{\xi}_{0}\right)^{-1} \frac{\partial \boldsymbol{\mu}(\boldsymbol{\xi})}{\partial[\boldsymbol{\xi}]_{k}}\right|_{\boldsymbol{\xi}=\boldsymbol{\xi}_{0}}\right\}$

$$
\forall i=1,2,3, k=1,2,3 \text {. }
$$

\section{B. The Unconditional Case}

In the unconditional model, the signal is assumed to be complex circular Gaussian (with zero mean and variance $\sigma_{s}^{2} \boldsymbol{I}$ ) independent from the noise. Consequently, the covariance parameterized observation model given by $\chi \sim \mathcal{C N}\left(\mathbf{0}, \boldsymbol{\Sigma}\left(\boldsymbol{\xi}_{0}\right)\right)$, in which

$$
\boldsymbol{\Sigma}\left(\boldsymbol{\xi}_{0}\right)=\sigma_{s}^{2}\left(\boldsymbol{e}\left(\omega_{0}\right) \otimes \boldsymbol{u}\left(\psi_{0}, \rho_{0}\right)\right)\left(\boldsymbol{e}\left(\omega_{0}\right) \otimes \boldsymbol{u}\left(\psi_{0}, \rho_{0}\right)\right)^{H}+\boldsymbol{\Sigma}_{\text {noise }}
$$


Then, by applying (3), one obtains

$$
\begin{gathered}
{\left[\operatorname{FIM}\left(\boldsymbol{\xi}_{0}\right)\right]_{i, k}=} \\
\operatorname{Ttr}\left\{\left.\left.\boldsymbol{\Sigma}\left(\boldsymbol{\xi}_{0}\right)^{-1} \frac{\partial \boldsymbol{\Sigma}(\boldsymbol{\xi})}{\partial[\boldsymbol{\xi}]_{i}}\right|_{\boldsymbol{\xi}=\boldsymbol{\xi}_{0}} \boldsymbol{\Sigma}\left(\boldsymbol{\xi}_{0}\right)^{-1} \frac{\partial \boldsymbol{\Sigma}(\boldsymbol{\xi})}{\partial[\boldsymbol{\xi}]_{k}}\right|_{\boldsymbol{\xi}=\boldsymbol{\xi}_{0}}\right\} \\
\forall i=1,2,3, k=1,2,3 .
\end{gathered}
$$

\section{DETERMINISTIC LOWER BOUNDS BACKGROUND AND DERIVATION}

The unification presented in [15] gives analytical expressions of the McAulay-Seidman bound (MSB, $C_{\mathrm{MSB}}^{(L)}$ ), the HammersleyChapman-Robbins bound (HCRB, $C_{\mathrm{HCRB}}^{(L)}$ ), the McAulayHofstetter bound (MHB, $C_{\mathrm{MHB}}^{(L)}$ ) and the Todros-Tabrikian Bound (TTB, $C_{\mathrm{TTB}}^{(L)}$ ). Specifically, we can notice that:

$$
\boldsymbol{C}_{\mathrm{MSB}}^{(L)}=\boldsymbol{\Phi} \boldsymbol{\Psi}^{-1} \boldsymbol{\Phi}^{T}
$$

where $\boldsymbol{\Phi}=\left[\boldsymbol{\xi}_{1}-\boldsymbol{\xi}_{0}, \ldots, \boldsymbol{\xi}_{L}-\boldsymbol{\xi}_{0}\right]$ in which $\boldsymbol{\xi}_{l}$ denotes the $l$-th test point for $l=1, \ldots, L$. Whereas, $[\boldsymbol{\Psi}]_{m, n}=$ $E_{\boldsymbol{\chi} \mid \boldsymbol{\xi}_{0}}\left\{\nu\left(\boldsymbol{\chi}, \boldsymbol{\xi}_{m}\right) \nu\left(\boldsymbol{\chi}, \boldsymbol{\xi}_{n}\right)\right\}$ for $m=1, \ldots, L, n=1, \ldots, L$ and $\nu\left(\chi, \boldsymbol{\xi}_{l}\right)$ denotes the ratio-likelihood function, given by $\nu\left(\boldsymbol{\chi}, \boldsymbol{\xi}_{l}\right)=\frac{p\left(\boldsymbol{\chi} \mid \boldsymbol{\xi}_{l}\right)}{p\left(\boldsymbol{\chi} \mid \boldsymbol{\xi}_{0}\right)}$. Furthermore, the HCRB and the MHB are given by

$$
\boldsymbol{C}_{\mathrm{HCRB}}^{(L)}=\boldsymbol{\Phi}\left(\boldsymbol{\Psi}-\mathbf{1}_{L} \mathbf{1}_{L}^{T}\right)^{-1} \boldsymbol{\Phi}^{T}
$$

and

$$
\boldsymbol{C}_{\mathrm{MHB}}^{(L)}=\boldsymbol{C}_{\mathrm{CRB}}+\boldsymbol{Q} \boldsymbol{R}^{-1} \boldsymbol{Q}^{T}
$$

where $\boldsymbol{C}_{\mathrm{CRB}}=\mathbf{F I M}^{-1}$ and $\boldsymbol{Q}=\boldsymbol{C}_{\mathrm{CRB}} \boldsymbol{D}-\boldsymbol{\Phi}$,

$$
\boldsymbol{R}=\boldsymbol{\Psi}-\boldsymbol{D}^{T} \boldsymbol{C}_{\mathrm{CRB}} \boldsymbol{D}
$$

in which $\boldsymbol{D}=\left[\boldsymbol{d}\left(\boldsymbol{\xi}_{1}\right), \ldots, \boldsymbol{d}\left(\boldsymbol{\xi}_{L}\right)\right]$, and each element of $\boldsymbol{D}$ is given by

$$
\boldsymbol{d}\left(\boldsymbol{\xi}_{l}\right)=\left(\left.\frac{\partial \operatorname{KLD}\left(p\left(\boldsymbol{\chi} \mid \boldsymbol{\xi}_{l}\right) \| p(\boldsymbol{\chi} \mid \boldsymbol{\xi})\right)}{\partial \boldsymbol{\xi}}\right|_{\boldsymbol{\xi}=\boldsymbol{\xi}_{0}}\right)^{T}
$$

The term $\operatorname{KLD}\left(p\left(\boldsymbol{\chi} \mid \boldsymbol{\xi}_{l}\right) \| p(\boldsymbol{\chi} \mid \boldsymbol{\xi})\right)$ denotes the Kullback-Leibler divergence [23] of $p(\boldsymbol{\chi} \mid \boldsymbol{\xi})$ from $p\left(\boldsymbol{\chi} \mid \boldsymbol{\xi}_{l}\right)$. Finally, the TTB is given by

$$
\boldsymbol{C}_{\mathrm{TTB}}^{(L, J)}=\boldsymbol{C}_{\mathrm{CRB}}+\boldsymbol{Q} \boldsymbol{W}^{H}\left(\boldsymbol{W} \boldsymbol{R} \boldsymbol{W}^{H}\right)^{-1} \boldsymbol{W} \boldsymbol{Q}^{T}
$$

in which, the three-dimensional-discrete-Fourier-transform (DFT) matrix is given by [15]

$$
[\boldsymbol{W}]_{i, l}=e^{-j \boldsymbol{\Omega}_{i}^{T} \boldsymbol{\xi}_{l}}
$$

in which $\Omega_{i}$ is expressed for the $i$-th frequency test bin by

$$
\boldsymbol{\Omega}_{i}^{T}=2 \pi\left[\frac{i_{\omega}}{\Delta_{\omega} L_{\omega}}, \frac{i_{\psi}}{\Delta_{\psi} L_{\psi}}, \frac{i_{\rho}}{\Delta_{\rho} L_{\rho}}\right],
$$

in which $L_{\omega}, L_{\psi}$ and $L_{\rho}$ represent the number of test points w.r.t. the unknown parameters $\omega, \psi$ and $\rho$, and $L=L_{\omega} L_{\psi} L_{\rho}$. The uniform inter-test points w.r.t. the unknown parameters are represented by $\omega, \psi$ and $\rho \Delta_{\omega}, \Delta_{\psi}$ and $\Delta_{\rho}$, respectively. Similarly, $i_{\omega}, i_{\psi}$ and $i_{\rho}$ denote the index of test-bin in the threedimensional of the frequency domain, $i_{\omega} \in 1, \ldots, L_{\omega}, i_{\psi} \in$ $1, \ldots, L_{\psi}$ and $i_{\rho} \in 1, \ldots, L_{\rho}$. Consequently, the index $i$ is a unique combination of $i_{\omega}, i_{\psi}, i_{\rho}$ where the total number of these combinations is denoted by $J$.

In the remaining of this section, we derive analytical expressions of the MSB, the HCRB, the MHB and the TTB for conditional and unconditional observation model.

\section{A. The Conditional Case}

Let us partition the FIM w.r.t. the signal parameter $\boldsymbol{\xi}_{0}$ as follow

$$
\boldsymbol{F I M}\left(\boldsymbol{\xi}_{0}\right)=\left[\begin{array}{lll}
F_{\omega, \omega} & F_{\omega, \psi} & F_{\omega, \rho} \\
F_{\psi, \omega} & F_{\psi, \psi} & F_{\psi, \rho} \\
F_{\rho, \omega} & F_{\rho, \psi} & F_{\rho, \rho}
\end{array}\right]
$$

in which, we used the notation $F_{u, v}$, where the lower script $u, v$ denotes the considered part of the FIM which corresponds to the derivation according to parameters $u$ and $v$ as shown in (3). Using (5), the entries of the FIM are given by

$$
\begin{aligned}
F_{\omega, \omega}= & 2 \Re\left[\left(\boldsymbol{s} \otimes\left(j \boldsymbol{e}^{\prime}\left(\omega_{0}\right) \otimes \boldsymbol{u}\left(\psi_{0}, \rho_{0}\right)\right)\right)^{H} \boldsymbol{\Sigma}_{\text {noise }}^{-1}\right. \\
& \left.\left(\boldsymbol{s} \otimes\left(j \boldsymbol{e}^{\prime}\left(\omega_{0}\right) \otimes \boldsymbol{u}\left(\psi_{0}, \rho_{0}\right)\right)\right)\right]
\end{aligned}
$$

$$
\begin{aligned}
F_{\psi, \psi}= & 2 \Re\left[\left.\left(\boldsymbol{s} \otimes\left(\boldsymbol{e}\left(\omega_{0}\right) \otimes \frac{\partial \boldsymbol{u}\left(\psi, \rho_{0}\right)}{\partial \psi}\right)\right)^{H}\right|_{\psi=\psi_{0}} \boldsymbol{\Sigma}_{\text {noise }}^{-1}\right. \\
& \left.\left.\left(\boldsymbol{s}^{H} \otimes\left(\boldsymbol{e}\left(\omega_{0}\right) \otimes \frac{\partial \boldsymbol{u}\left(\psi, \rho_{0}\right)}{\partial \psi}\right)\right)\right|_{\psi=\psi_{0}}\right]
\end{aligned}
$$

and

$$
\begin{aligned}
F_{\rho, \rho}= & 2 \Re\left[\left.\left(\boldsymbol{s}^{H} \otimes\left(\boldsymbol{e}\left(\omega_{0}\right) \otimes \frac{\partial \boldsymbol{u}\left(\psi_{0}, \rho\right)}{\partial \rho}\right)^{H}\right)\right|_{\rho=\rho_{0}} \boldsymbol{\Sigma}_{\text {noise }}^{-1}\right. \\
& \left.\left.\left(\boldsymbol{s} \otimes\left(\boldsymbol{e}\left(\omega_{0}\right) \otimes \frac{\partial \boldsymbol{u}\left(\psi_{0}, \rho\right)}{\partial \rho}\right)\right)\right|_{\rho=\rho_{0}}\right]
\end{aligned}
$$

in which $e^{\prime}\left(\omega_{0}\right)=\left[d_{1} e^{j d_{1} \omega_{0}}, \ldots, d_{N} e^{j d_{N} \omega_{0}}\right]^{T}$. The cross terms are given by

$$
\begin{gathered}
F_{\psi, \rho}=F_{\rho, \psi}=2 \Re\left[\left.\left(\boldsymbol{s} \otimes\left(\boldsymbol{e}\left(\omega_{0}\right) \otimes \frac{\partial \boldsymbol{u}\left(\psi, \rho_{0}\right)}{\partial \psi}\right)\right)^{H}\right|_{\psi=\psi_{0}} \boldsymbol{\Sigma}_{\text {noise }}^{-1}\right. \\
\left.\left.\left(\boldsymbol{s} \otimes\left(\boldsymbol{e}\left(\omega_{0}\right) \otimes \frac{\partial \boldsymbol{u}\left(\psi_{0}, \rho\right)}{\partial \rho}\right)\right)\right|_{\rho=\rho_{0}}\right] \\
F_{\omega, \rho}=F_{\rho, \omega}=2 \Re\left[\left(\boldsymbol{s} \otimes\left(j \boldsymbol{e}^{\prime}\left(\omega_{0}\right) \otimes \boldsymbol{u}\left(\psi_{0}, \rho_{0}\right)\right)\right)^{H} \boldsymbol{\Sigma}_{\text {noise }}^{-1}\right. \\
\left.\left.\left(\boldsymbol{s} \otimes\left(\boldsymbol{e}\left(\omega_{0}\right) \otimes \frac{\partial \boldsymbol{u}\left(\psi_{0}, \rho\right)}{\partial \rho}\right)\right)\right|_{\rho=\rho_{0}}\right]
\end{gathered}
$$

and

$$
\begin{aligned}
F_{\omega, \psi}=F_{\psi, \omega}= & 2 \Re\left[\left(\boldsymbol{s} \otimes\left(j \boldsymbol{e}^{\prime}\left(\omega_{0}\right) \otimes \boldsymbol{u}\left(\psi_{0}, \rho_{0}\right)\right)\right)^{H} \boldsymbol{\Sigma}_{\text {noise }}^{-1}\right. \\
& \left.\left.\left(\boldsymbol{s} \otimes\left(\boldsymbol{e}\left(\omega_{0}\right) \otimes \frac{\partial \boldsymbol{u}\left(\psi_{0}, \rho\right)}{\partial \rho}\right)\right)\right|_{\rho=\rho_{0}}\right]
\end{aligned}
$$

In particular, if $\boldsymbol{n}_{\mathrm{n}}(t)$ is a complex circular white Gaussian noise with zero-mean and unknown variance $\sigma_{n}^{2}$, assumed to be uncorrelated both temporally and spatially (i.e., $\left.\boldsymbol{\Sigma}_{\text {noise }}=\sigma_{n}^{2} \boldsymbol{I}_{2 N T}\right)$. The above expressions simplifies to $F_{\omega, \omega}=\frac{2}{\sigma_{n}^{2}}\left\|\boldsymbol{d}_{N}\right\|_{2}^{2}\|\boldsymbol{a}\|_{2}^{2}, \quad F_{\psi, \psi}=\frac{2 N}{\sigma_{n}^{2}} \sin \left(\rho_{0}\right)^{2}\|\boldsymbol{a}\|_{2}^{2}$ and $F_{\rho, \rho}=\frac{2 N}{\sigma_{n}^{2}}\|\boldsymbol{a}\|_{2}^{2}$. Whereas, the cross terms are given by $F_{\omega, \psi}=F_{\psi, \omega}=\frac{2}{\sigma_{n}^{2}} \sin \left(\rho_{0}\right)^{2}\left\|\boldsymbol{d}_{N}\right\|_{1}^{2}\|\boldsymbol{a}\|_{2}^{2}$ and $F_{\omega, \rho}=F_{\rho, \omega}=$ $F_{\rho, \psi}=F_{\psi, \rho}=0$ with $\boldsymbol{a}=\boldsymbol{s}$ and $\boldsymbol{d}_{N}=\left[d_{1}, d_{2}, \ldots, d_{n}\right]^{T}$. 
Considering an identifiable situation in which $|\mathbf{F I M}| \neq 0$, one obtains after some calculus (the general case, i.e., $2 \pi \frac{A_{s l}}{\lambda} \neq L_{s d}$, is given in the Appendix)

$$
\begin{gathered}
\mathbf{C R B}\left(\boldsymbol{\xi}_{0}\right)=\frac{\sigma_{n}^{2}}{2\|\boldsymbol{a}\|_{2}^{2}} \\
{\left[\begin{array}{c}
\frac{N}{N\left\|\boldsymbol{d}_{N}\right\|_{2}^{2}-\sin \left(\rho_{0}\right)^{2}\left\|\boldsymbol{d}_{N}\right\|_{1}^{4}} \\
-\frac{\left\|\boldsymbol{d}_{N}\right\|_{1}^{2}}{N\left\|\boldsymbol{d}_{N}\right\|_{2}^{2}-\sin \left(\rho_{0}\right)^{2}\left\|\boldsymbol{d}_{N}\right\|_{1}^{4}} \\
0
\end{array}\right.}
\end{gathered}
$$

On the other hand, in (12) the KLD for conditional case is given by

$$
\begin{aligned}
& \operatorname{KLD}\left(p\left(\boldsymbol{\chi} \mid \boldsymbol{\xi}_{l}\right) \| p(\chi \mid \boldsymbol{\xi})\right)=\int p\left(\chi \mid \boldsymbol{\xi}_{l}\right) \ln \left(\frac{p\left(\boldsymbol{\chi} \mid \boldsymbol{\xi}_{l}\right)}{p(\boldsymbol{\chi} \mid \boldsymbol{\xi})}\right) d \boldsymbol{\chi} \\
& =\left(\boldsymbol{\mu}(\boldsymbol{\xi})-\boldsymbol{\mu}\left(\boldsymbol{\xi}_{l}\right)\right)^{H} \boldsymbol{\Sigma}_{\text {noise }}^{-1}\left(\boldsymbol{\mu}\left(\boldsymbol{\xi}_{l}\right)-\boldsymbol{\mu}(\boldsymbol{\xi})\right)
\end{aligned}
$$

thus,

$$
\begin{aligned}
\boldsymbol{d}\left(\boldsymbol{\xi}_{l}\right) & =-\left.\left(\frac{\partial \operatorname{KLD}\left(p\left(\boldsymbol{\chi} \mid \boldsymbol{\xi}_{l}\right)|| p(\boldsymbol{\chi} \mid \boldsymbol{\xi})\right)}{\partial \boldsymbol{\xi}}\right)^{T}\right|_{\boldsymbol{\xi}=\boldsymbol{\xi}_{0}} \\
& =-\left.2 \Re\left\{\left(\boldsymbol{\mu}(\boldsymbol{\xi})-\boldsymbol{\mu}\left(\boldsymbol{\xi}_{l}\right)\right)^{H} \boldsymbol{\Sigma}_{\text {noise }}^{-1} \frac{\partial \boldsymbol{\mu}(\boldsymbol{\xi})^{H}}{\partial \boldsymbol{\xi}}\right\}^{T}\right|_{\boldsymbol{\xi}=\boldsymbol{\xi}_{0}}
\end{aligned}
$$

Using, (11), each element of $\boldsymbol{\Psi}$ is of the form

$$
\begin{aligned}
{[\boldsymbol{\Psi}]_{m, n} } & =E_{\boldsymbol{\chi} \mid \boldsymbol{\xi}_{0}}\left\{\boldsymbol{\nu}\left(\boldsymbol{\chi}, \boldsymbol{\xi}_{m}\right) \boldsymbol{\nu}\left(\boldsymbol{\chi}, \boldsymbol{\xi}_{n}\right)\right\} \\
& =\frac{1}{\pi^{2 N T}\left|\boldsymbol{\Sigma}_{\text {noise }}\right|} \int e^{\kappa_{1}} \\
& =\alpha\left(\boldsymbol{\xi}_{m}, \boldsymbol{\xi}_{n}\right) \int \frac{1}{\pi^{2 N T}\left|\boldsymbol{\Sigma}_{\text {noise }}\right|} e^{\kappa_{2}} d \boldsymbol{\chi} \\
& =\alpha\left(\boldsymbol{\xi}_{m}, \boldsymbol{\xi}_{n}\right)
\end{aligned}
$$

in which

$$
\begin{aligned}
\kappa_{1}= & -\left(\boldsymbol{\chi}-\boldsymbol{\mu}\left(\boldsymbol{\xi}_{m}\right)\right)^{H} \boldsymbol{\Sigma}_{\text {noise }}^{-1}\left(\boldsymbol{\chi}-\boldsymbol{\mu}\left(\boldsymbol{\xi}_{m}\right)\right) \\
& -\left(\boldsymbol{\chi}-\boldsymbol{\mu}\left(\boldsymbol{\xi}_{n}\right)\right)^{H} \boldsymbol{\Sigma}_{\text {noise }}^{-1}\left(\boldsymbol{\chi}-\boldsymbol{\mu}\left(\boldsymbol{\xi}_{n}\right)\right)+\left(\boldsymbol{\chi}-\boldsymbol{\mu}\left(\boldsymbol{\xi}_{0}\right)\right)^{H} \\
& \boldsymbol{\Sigma}_{\text {noise }}^{-1}\left(\boldsymbol{\chi}-\boldsymbol{\mu}\left(\boldsymbol{\xi}_{0}\right)\right) d \boldsymbol{\chi}
\end{aligned}
$$

and

$$
\begin{array}{r}
\kappa_{2}=-\left(\boldsymbol{\chi}-\boldsymbol{\mu}\left(\boldsymbol{\xi}_{m}\right)-\boldsymbol{\mu}\left(\boldsymbol{\xi}_{n}\right)+\boldsymbol{\mu}\left(\boldsymbol{\xi}_{0}\right)\right)^{H} \\
\boldsymbol{\Sigma}_{\text {noise }}^{-1}\left(\boldsymbol{\chi}-\boldsymbol{\mu}\left(\boldsymbol{\xi}_{m}\right)-\boldsymbol{\mu}\left(\boldsymbol{\xi}_{n}\right)+\boldsymbol{\mu}\left(\boldsymbol{\xi}_{0}\right)\right)
\end{array}
$$

and

$$
\alpha\left(\boldsymbol{\xi}_{m}, \boldsymbol{\xi}_{n}\right)=e^{2 \Re\left\{\left(\boldsymbol{\mu}\left(\boldsymbol{\xi}_{m}\right)-\boldsymbol{\mu}\left(\boldsymbol{\xi}_{0}\right)\right)^{H} \boldsymbol{\Sigma}_{\text {noise }}^{-1}\left(\boldsymbol{\mu}\left(\boldsymbol{\xi}_{n}\right)-\boldsymbol{\mu}\left(\boldsymbol{\xi}_{0}\right)\right)\right\}}
$$

At last, plugging (4), (16)-(20) into (8)-(10) and (13) one obtains $C_{\mathrm{C}-\mathrm{MSB}}^{(L)}, \boldsymbol{C}_{\mathrm{C}-\mathrm{HCRB}}^{(L)}, \boldsymbol{C}_{\mathrm{C}-\mathrm{MHB}}^{(L)}$ and $\boldsymbol{C}_{\mathrm{C}-\mathrm{TTB}}^{(L, J)}$, in which $\mathrm{C}$ stands stands for Conditional.

\section{B. The Unconditional Case}

Let us consider the unconditional model. For simplicity we define $\boldsymbol{\Gamma}\left(\boldsymbol{\xi}_{0}\right)=\boldsymbol{e}\left(\omega_{0}\right) \otimes \boldsymbol{u}\left(\psi_{0}, \rho_{0}\right)$ and let us recall the following matrix properties $\operatorname{Tr}(\boldsymbol{X} \boldsymbol{Y})=\operatorname{vec}\left(\boldsymbol{X}^{H}\right)^{H} \operatorname{vec}(\boldsymbol{Y})$,
$\operatorname{vec}(\boldsymbol{X} \boldsymbol{Y} \boldsymbol{Z})=\left(\boldsymbol{Z}^{T} \otimes \boldsymbol{X}\right) \operatorname{vec}(\boldsymbol{Y})$, which hold for any matrices $\boldsymbol{X}, \boldsymbol{Y}$ and $\boldsymbol{Z}$ [24]. Using these properties along with (7), we obtain

$$
\begin{aligned}
\frac{1}{T} \boldsymbol{F I M}\left(\boldsymbol{\xi}_{0}\right)= & \left.\left(\frac{\partial \boldsymbol{r}}{\partial \boldsymbol{\xi}^{T}}\right)^{H}\right|_{\boldsymbol{\xi}=\boldsymbol{\xi}_{0}}\left(\boldsymbol{\Sigma}\left(\boldsymbol{\xi}_{0}\right)^{-T} \otimes\right. \\
& \left.\boldsymbol{\Sigma}\left(\boldsymbol{\xi}_{0}\right)^{-1}\right)\left.\left(\frac{\partial \boldsymbol{r}}{\partial \boldsymbol{\xi}^{T}}\right)\right|_{\boldsymbol{\xi}=\boldsymbol{\xi}_{0}} \\
= & {\left[\begin{array}{lll}
\boldsymbol{g}_{\omega}^{H} \boldsymbol{g}_{\omega} & \boldsymbol{g}_{\omega}^{H} \boldsymbol{g}_{\psi} & \boldsymbol{g}_{\omega}^{H} \boldsymbol{g}_{\rho} \\
\boldsymbol{g}_{\psi}^{H} \boldsymbol{g}_{\omega} & \boldsymbol{g}_{\psi}^{H} \boldsymbol{g}_{\psi} & \boldsymbol{g}_{\psi}^{H} \boldsymbol{g}_{\rho} \\
\boldsymbol{g}_{\rho}^{H} \boldsymbol{g}_{\omega} & \boldsymbol{g}_{\rho}^{H} \boldsymbol{g}_{\psi} & \boldsymbol{g}_{\rho}^{H} \boldsymbol{g}_{\rho}
\end{array}\right] }
\end{aligned}
$$

where

$$
\boldsymbol{r}=\operatorname{vec}\left(\boldsymbol{\Sigma}\left(\boldsymbol{\xi}_{0}\right)\right)=\sigma_{s}^{2}\left(\boldsymbol{\Gamma}^{c}\left(\boldsymbol{\xi}_{0}\right) \otimes \boldsymbol{\Gamma}\left(\boldsymbol{\xi}_{0}\right)\right)+\operatorname{vec}\left(\boldsymbol{\Sigma}_{\text {noise }}\right)
$$

and

$$
\boldsymbol{g}_{\omega}=\operatorname{vec}\left(\left.\boldsymbol{\Sigma}\left(\boldsymbol{\xi}_{0}\right)^{-\frac{1}{2}} \frac{\partial \boldsymbol{\Sigma}(\boldsymbol{\xi})}{\partial \omega}\right|_{\boldsymbol{\xi}=\boldsymbol{\xi}_{0}} \boldsymbol{\Sigma}\left(\boldsymbol{\xi}_{0}\right)^{-\frac{1}{2}}\right)
$$

If the sensor noise is both spatially and temporally white, as $\boldsymbol{\Sigma}_{\text {noise }}=\sigma_{n}^{2} \boldsymbol{I}_{2 N}$, using the matrix inversion lemma [25] into (6), one can obtain

$$
\boldsymbol{\Sigma}\left(\boldsymbol{\xi}_{0}\right)^{-1}=\frac{1}{\sigma_{n}^{2}} \boldsymbol{I}_{2 N}-\frac{\sigma_{s}^{2}}{\sigma_{n}^{4}+N \sigma_{s}^{2} \sigma_{n}^{2}} \boldsymbol{\Gamma}\left(\boldsymbol{\xi}_{0}\right) \boldsymbol{\Gamma}^{H}\left(\boldsymbol{\xi}_{0}\right)
$$

On the other hand, in (12) the KLD is obtained $\operatorname{KLD}\left(p\left(\boldsymbol{\chi} \mid \boldsymbol{\xi}_{l}\right) \| p(\boldsymbol{\chi} \mid \boldsymbol{\xi})\right)=E_{\boldsymbol{\chi} \mid \boldsymbol{\xi}_{l}}\left\{\boldsymbol{\chi}^{H}\left(\boldsymbol{I}_{T} \otimes \boldsymbol{\Sigma}(\boldsymbol{\xi})\right)^{-1} \boldsymbol{\chi}\right\}-$ $E_{\boldsymbol{\chi} \mid \boldsymbol{\xi}_{l}}\left\{\boldsymbol{\chi}^{H}\left(\boldsymbol{I}_{T} \otimes \boldsymbol{\Sigma}\left(\boldsymbol{\xi}_{l}\right)\right)^{-1} \boldsymbol{\chi}\right\}^{+}+T \ln |\boldsymbol{\Sigma}(\boldsymbol{\xi})|-$ $T \ln \left|\boldsymbol{\Sigma}\left(\boldsymbol{\xi}_{l}\right)\right|$ in which $E_{\boldsymbol{\chi} \mid \boldsymbol{\xi}_{l}}\left\{\boldsymbol{\chi}^{H}\left(\boldsymbol{I}_{T} \otimes \boldsymbol{\Sigma}(\boldsymbol{\xi})\right)^{-1} \boldsymbol{\chi}\right\}=$ $\sum_{i=1}^{2 N T} \sum_{j=1}^{2 N T} E_{\boldsymbol{\chi} \mid \boldsymbol{\xi}_{l}}\left\{[\boldsymbol{\chi}]_{i}^{c}[\boldsymbol{\chi}]_{j}^{c}\left[\left(\boldsymbol{I}_{T} \otimes \boldsymbol{\Sigma}(\boldsymbol{\xi})\right)^{-1}\right]_{i, j}\right\}=$ $\operatorname{Ttr}\left\{\boldsymbol{\Sigma}\left(\boldsymbol{\xi}_{l}\right) \boldsymbol{\Sigma}(\boldsymbol{\xi})^{-1}\right\}$ and similarly, one obtains $E_{\boldsymbol{\chi} \mid \boldsymbol{\xi}_{l}}\left\{\chi^{H} \boldsymbol{\Sigma}\left(\boldsymbol{\xi}_{l}\right)^{-1} \boldsymbol{\xi}\right\}=2 N T$. Consequently,

$$
\begin{aligned}
\operatorname{KLD}\left(p\left(\boldsymbol{\chi} \mid \boldsymbol{\xi}_{l}\right)|| p(\boldsymbol{\chi} \mid \boldsymbol{\xi})\right) & =\operatorname{Ttr}\left(\boldsymbol{\Sigma}\left(\boldsymbol{\xi}_{l}\right) \boldsymbol{\Sigma}(\boldsymbol{\xi})^{-1}\right)-2 N T \\
& +T \ln \left(\frac{\left|\boldsymbol{\Sigma}\left(\boldsymbol{\xi}_{l}\right)\right|}{|\boldsymbol{\Sigma}(\boldsymbol{\xi})|}\right)
\end{aligned}
$$

In addition, the $l$-th member of $\boldsymbol{D}$ in (12) is given by

$$
\begin{aligned}
& \frac{\partial \operatorname{KLD}\left(p\left(\boldsymbol{\chi} \mid \boldsymbol{\xi}_{l}\right)|| p(\boldsymbol{\chi} \mid \boldsymbol{\xi})\right)}{\partial \boldsymbol{\xi}}=\operatorname{Ttr}\left\{\boldsymbol{\Sigma}(\boldsymbol{\xi})^{-1} \frac{\partial \boldsymbol{\Sigma}(\boldsymbol{\xi})}{\partial \boldsymbol{\xi}}\right\} \\
& +\operatorname{Ttr}\left\{-\boldsymbol{\Sigma}\left(\boldsymbol{\xi}_{l}\right) \boldsymbol{\Sigma}(\boldsymbol{\xi})^{-1} \frac{\partial \boldsymbol{\Sigma}(\boldsymbol{\xi})}{\partial \boldsymbol{\xi}} \boldsymbol{\Sigma}(\boldsymbol{\xi})^{-1}\right\}
\end{aligned}
$$

and from (11), the element of $\boldsymbol{\Psi}$ is

$$
\begin{aligned}
{[\boldsymbol{\Psi}]_{m, n} } & =E_{\boldsymbol{\chi} \mid \boldsymbol{\xi}_{0}}\left\{\boldsymbol{\nu}\left(\boldsymbol{\chi}, \boldsymbol{\xi}_{m}\right) \boldsymbol{\nu}\left(\boldsymbol{\chi}, \boldsymbol{\xi}_{n}\right)\right\} \\
& =\frac{\left|\boldsymbol{I}_{T} \otimes \boldsymbol{\Sigma}\left(\boldsymbol{\xi}_{0}\right)\right|^{2}}{\left|\boldsymbol{I}_{T} \otimes \boldsymbol{\Sigma}\left(\boldsymbol{\xi}_{m}\right)\right|\left|\boldsymbol{I}_{T} \otimes \boldsymbol{\Sigma}\left(\boldsymbol{\xi}_{n}\right)\right|} \int e^{\kappa 3} p\left(\boldsymbol{\chi} \mid \boldsymbol{\xi}_{0}\right) d \boldsymbol{\chi} \\
& =\frac{\left|\boldsymbol{\Sigma}\left(\boldsymbol{\xi}_{0}\right)\right|\left|\boldsymbol{\Sigma}\left(\boldsymbol{\xi}_{m}\right)^{-1}+\boldsymbol{\Sigma}\left(\boldsymbol{\xi}_{n}\right)^{-1}-\boldsymbol{\Sigma}\left(\boldsymbol{\xi}_{0}\right)^{-1}\right|}{\left|\boldsymbol{\Sigma}\left(\boldsymbol{\xi}_{m}\right)\right|\left|\boldsymbol{\Sigma}\left(\boldsymbol{\xi}_{n}\right)\right|}
\end{aligned}
$$

with

$$
\begin{array}{r}
\kappa_{3}=-\boldsymbol{\chi}^{H}\left(\left(\boldsymbol{I}_{T} \otimes \boldsymbol{\Sigma} \boldsymbol{\xi}_{m}\right)\right)^{-1}+\left(\boldsymbol{I}_{T} \otimes\right. \\
\left.\left.\boldsymbol{\Sigma}\left(\boldsymbol{\xi}_{n}\right)\right)^{-1}-2\left(\boldsymbol{I}_{T} \otimes \boldsymbol{\Sigma}\left(\boldsymbol{\xi}_{0}\right)\right)^{-1}\right) \boldsymbol{\chi}
\end{array}
$$

Finally, $\boldsymbol{C}_{\mathrm{U}-\mathrm{MSB}}^{(L)}, \boldsymbol{C}_{\mathrm{U}-\mathrm{HCRB}}^{(L)}, \boldsymbol{C}_{\mathrm{U}-\mathrm{MHB}}^{(L)}$, and $\boldsymbol{C}_{\mathrm{U}-\mathrm{TTB}}^{(L, J)}$ are given by replacing (6), (21) - (23) into (8), (9), (10) and (13), in which U stands stands for Unconditional. 


\section{NUMERICAL INVESTIGATION}

Numerical results are presented in this section for a non-uniform linear COLD array with $N=8$ sensors. One narrowband farfield source is located according to $\theta_{0}=60^{\circ}$ and the polarization state parameters are given by $\rho_{0}=30^{\circ}$ and $\psi_{0}=45^{\circ}$. Simulations are performed for $T=15$ snapshots.

\section{A. Analytical and numerical analysis of the derived lower bounds}

The aim of this part is to examine the usefulness of $C_{\mathrm{MSB}}^{(L)}$, $\boldsymbol{C}_{\mathrm{HCRB}}^{(L)}, \boldsymbol{C}_{\mathrm{MHB}}^{(L)}$ and $\boldsymbol{C}_{\mathrm{TTB}}^{(L, J)}$ to predict the SNR threshold. Using, (10), (11), (13) and (17), we plot the derived lower bounds for the parameter $\omega_{0}$ for both conditional and unconditional models in Fig.2 and Fig.3, respectively. These figures show that the derived bounds exhibit a threshold effect around $-9 \mathrm{~dB}$. We note also that the new proposed TTB provides a better prediction of the SNR threshold as expected. Furthermore, for $J<L$, the computational cost of the TTB is lower in comparison to the MSB, HCRB and MHB. This is mainly due to the inversion of a $J \times J$ matrix due to presence of the discrete transform matrix $\boldsymbol{W}$ instead of the initial $L \times L$ matrix inversion.

In Fig. 4 and 5, we plot the TTB w.r.t. $\omega$ vs. SNR for different polarization parameters $\psi_{0}$ and $\rho_{0}$ in the conditonal case (the same conclusion are noticed for the unconditional case). From Fig.4, we notice that, no matter how $\psi_{0}$ changes, the breakdown point is approximately fixed for a given $\omega_{0}$, meaning that the effect of the polarisation state parameter $\psi$ can be neglected in designing the COLD array. In Fig.5 we focus on the effect of $\rho$, in which, this figure shows that, for a different value of polarization parameter $\rho$ and for a fixed SNR, the higher the $\rho_{0}$ is, the better are the performances (w.r.t. the MSE in the asymptotic region but also breakdown point).

\section{B. Frequency test-bins and their effect on the TTB}

One notes that increasing the number of sensors or test-points may improve the tightness of the bounds. This is also same for frequency-bins. However, this improvement comes at the expense of computational complexity. Considering there is an intuitive link between the performance of threshold prediction and the sequence of frequency test-bins. We give a sub-optimal method to design an optimal index of frequency test-bins in order to overcome an exhaustive search by computer over all possibilities. First, place only one frequency test-bin by minimizing the threshold SNR with respect to $J$ positions. Second, iterate the first step by placing the $n$-th frequency test-bin at once sequentially until $n=L$ with respect to $J-n+1$ remaining positions. To illustrate and compare the accuracy and usefulness of the aforementioned approach, two numerical examples for TTB are obtained: case 1- With the same number of test-points $L=32$, one notice that our sub-optimal method yields a very good agreement when compared with the searching solution performed by computer, as shown in Fig.6. Furthermore, this figure also shows us that increasing the number of frequency test-bins gives a considerable improvement in the SNR threshold prediction; case 2- In Fig.7, with the same number of test-points and frequency test-bins $L=J=32$, the threshold prediction of optimal index is nearly $1 \mathrm{~dB}$ better than the sequential one, which means that the index of frequency test-bins has an important effect on minimizing the ambiguity region threshold.

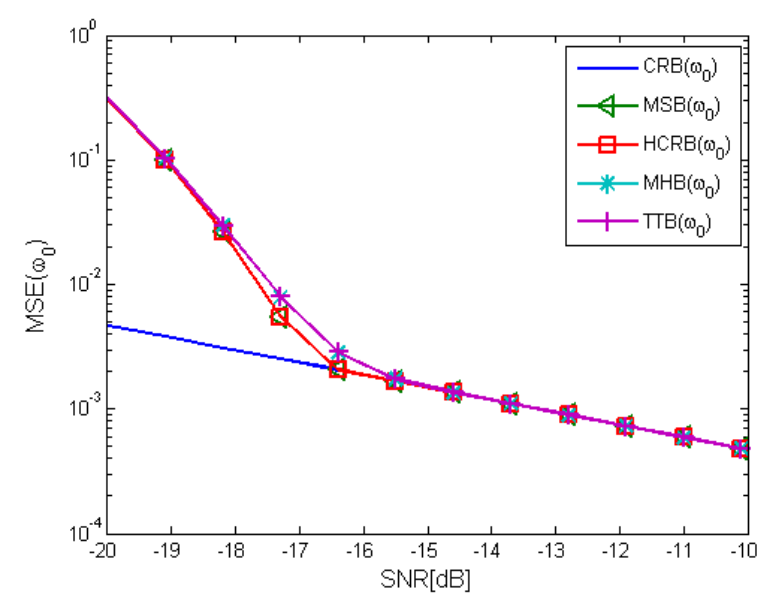

Fig. 2: Lower bounds on the mean square error (conditional case) w.r.t. $\omega$ for NULA-COLD array $(L=J=8)$.

As the number of snapshots $T$ increases, this advantage will be more apparent. Consequently, we notice that the proposed method with low complexity is useful for a threshold prediction problem, especially for the sensor arrays with large test-points.

\section{Designing a COLD array : Resolution factor vs threshold SNR}

To better comprehend the system performance w.r.t the resolution factor (RF) and the threshold SNR as a function of array geometry we consider Fig. 8 in which we compare all possible configuration for $N=8$ and a given aperture $A=23$ (more precisely, such context gives 74613 possible sensor array configurations). The term resolution factor is the minus curvature of the magnitude squared of the beampattern at the peak of its mainlobe and is determined by the array geometry [26] as:

$$
R F=\frac{8 d^{2} \pi^{2}}{N^{2} \lambda^{2}} \boldsymbol{d}_{N}^{T}\left(N \boldsymbol{I}_{N}-\mathbf{1}_{N}\right) \boldsymbol{d}_{N}
$$

in which $\boldsymbol{d}_{N}=\left[d_{1}, \ldots, d_{N}\right]^{T}$ denotes the $N \times 1$ vector of array element index locations.

In general, we would like the threshold SNR to be as small as possible and the RF to be as large as possible [26]. We see that the best performance is achieved at the upper-left corner of the distribution given in Fig.8, where there is a tradeoff between resolution and threshold prediction.

As an example, eight types of array configuration are considered, as shown in Table 1. From comparison of simulation results, one can further notice that: 1) For the same array aperture and same number of sensors, the SNR threshold prediction and resolution factor are greatly affected by the array geometric configuration.

2) The configuration that puts two sensors at the extremity and the rest in the middle has the worst performance for resolution factor; Contrary, places two average parts of sensors on both sides seems to be the best geometry configuration (at least, it is the case for $N=8$ and $A=23$ sensors). From example, Type 1 to Type 4 arrays, which have the good performance for the threshold SNR, are nearly $3 \mathrm{~dB}$ better than the relatively poor performance of type 7 and type 8 , where this is due to the sensor geometry configuration. 


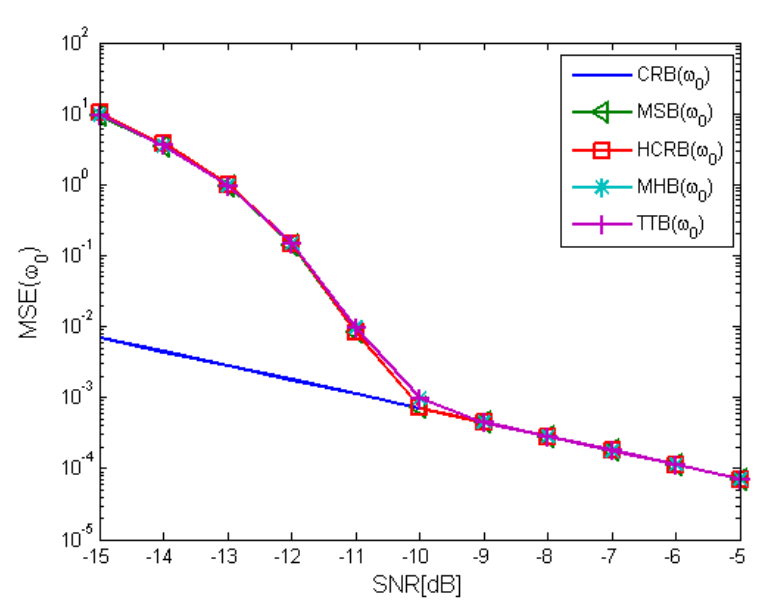

Fig. 3: Lower bounds on the mean square error (unconditional case) w.r.t. $\omega$ for NULA-COLD array $(L=J=8)$.

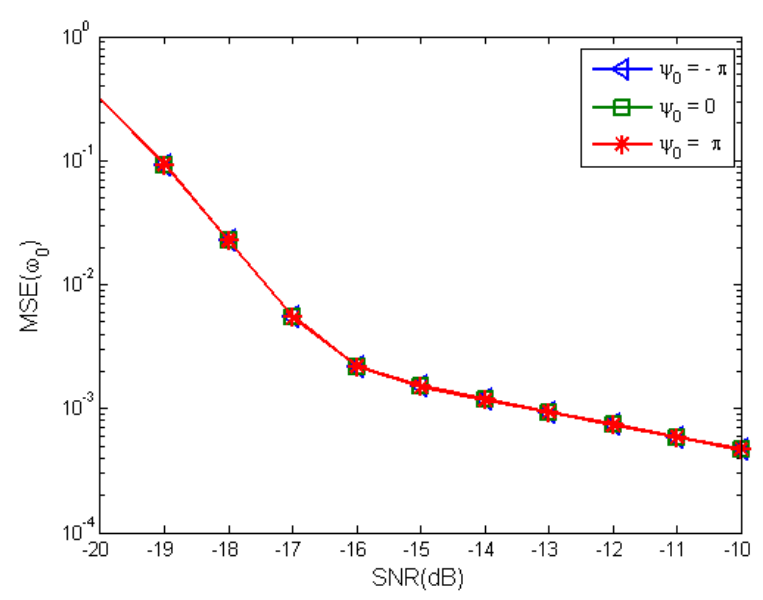

Fig. 4: TTB on the mean square error (conditional case) w.r.t. $\psi$ for NULA-COLD array. $(L=J=8)$

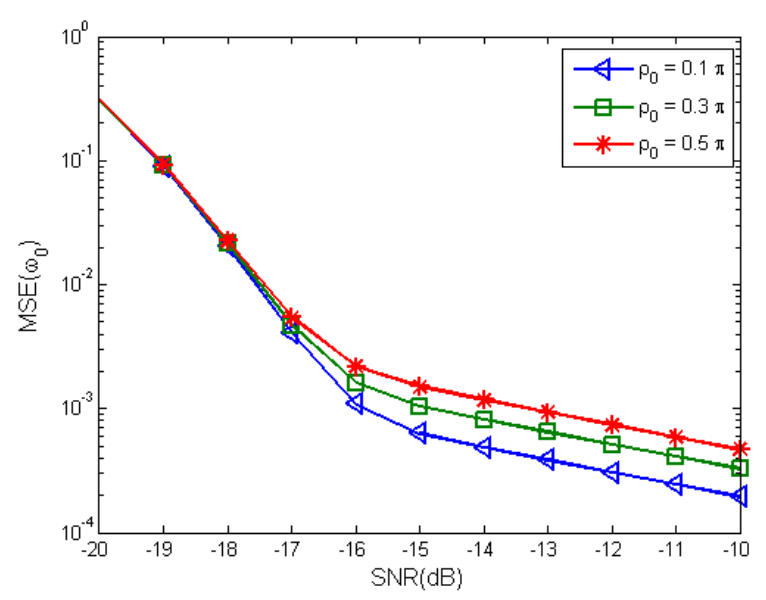

Fig. 5: TTB on the mean square error (conditionalc case) w.r.t. $\rho$ for NULA-COLD array. $(L=J=8)$

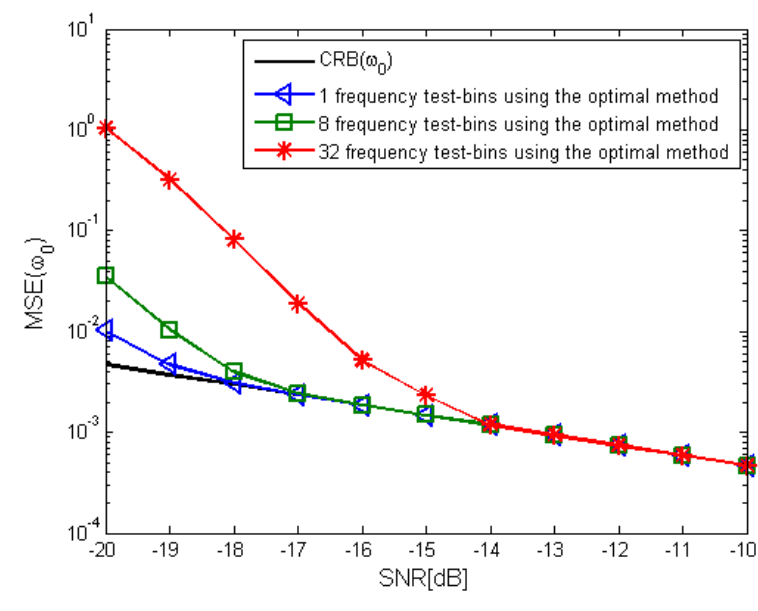

Fig. 6: Comparisons of different number of frequency test-bins for TTB threshold prediction with test-points $L=32$.

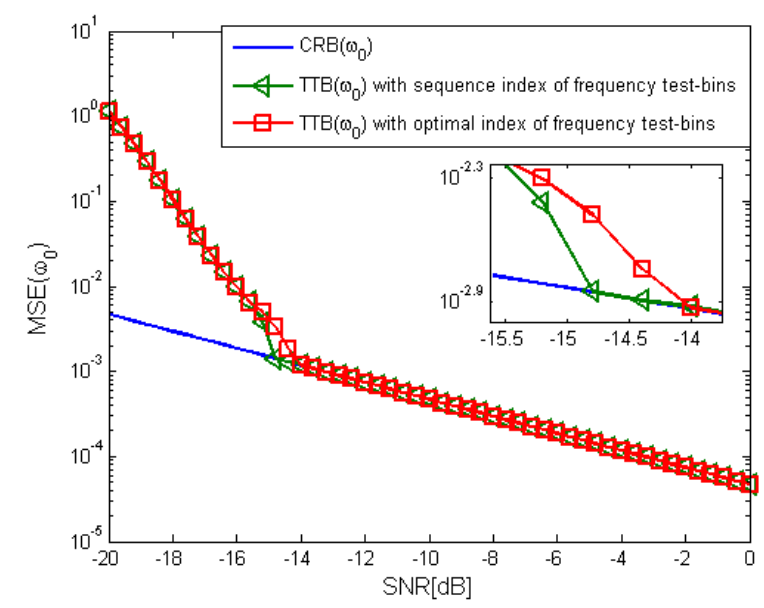

Fig. 7: Comparisons of different index of frequency test-bins for TTB threshold prediction with test-points $L=32$, frequency test-bins $J=32$.

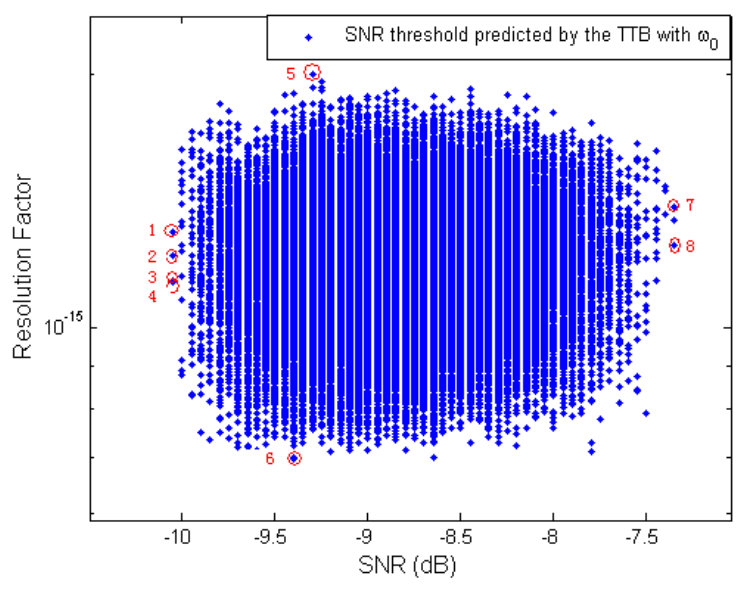

Fig. 8: Comparisons of different array geometric configurations for resolution factor w.r.t. SNR threshold predicted by the TTB (conditional case: $N=8, A=23$ ). 


\begin{tabular}{|c|c|c|c|}
\hline Array Type & Geometric Configuration & Resolution Factor & Threshold SNR \\
\hline Type 1 & $\bullet \bullet \circ \circ \circ \bullet \circ \circ \circ \bullet \circ \circ \circ \bullet \circ \bullet \circ \circ \circ \circ \circ \bullet \circ \bullet$ & & among the best configuration \\
\hline Type 2 & $\bullet \circ \bullet \circ \bullet \circ \circ \circ \circ \circ \bullet \circ \bullet \circ \circ \bullet \circ \circ \circ \circ \bullet \circ \circ \bullet$ & & among the best configuration \\
\hline Type 3 & $\bullet \circ \bullet \circ \bullet \circ \circ \circ \circ \circ \bullet \circ \bullet \circ \circ \bullet \circ \circ \bullet \circ \circ \circ \circ \bullet$ & & the best configuration \\
\hline Type 4 & $\bullet \bullet \circ \circ \circ \bullet \circ \circ \circ \bullet \circ \circ \circ \bullet \circ \bullet \circ \bullet \circ \circ \circ \circ \circ \bullet$ & & among the best configuration \\
\hline Type 5 & $\bullet \bullet \bullet \bullet \circ \circ \circ \circ \circ \circ \circ \circ \circ \circ \circ \circ \circ \circ \circ \circ \bullet \bullet \bullet \bullet$ & the best configuration & \\
\hline Type 6 & $\bullet \circ \circ \circ \circ \circ \circ \circ \circ \bullet \bullet \bullet \bullet \bullet \bullet \circ \circ \circ \circ \circ \circ \circ \circ \bullet$ & the worst configuration & \\
\hline Type 7 & $\bullet \bullet \circ \circ \circ \circ \circ \bullet \circ \circ \circ \circ \circ \circ \bullet \circ \bullet \circ \bullet \circ \circ \circ \bullet \bullet$ & & among the worst configuration \\
\hline Type 8 & $\bullet \circ \bullet \circ \circ \circ \circ \bullet \circ \circ \circ \bullet \circ \bullet \circ \circ \bullet \circ \circ \circ \circ \circ \bullet \bullet$ & & among the worst configuration \\
\hline
\end{tabular}

Table 1. Different array geometric configurations among the 74613 possibilities for $N=8$ and $A=23(\bullet$ and $\circ$ represent the position of sensor and missing sensors, respectively.)

\section{CONCLUSION}

In this paper, we derive explicit closed-form expressions of different deterministic lower bounds on the mean square error for the so-called non-uniform linear co-centered orthogonal loop and dipole arrays in a passive polarization source localization context. Taking advantage of these expressions, we analyse and characterize the performances in the asymptotic region and non-asymptotic region in terms of breakdown point prediction for the conditional and unconditional observation models. Finally, numerical simulations show the effect of each polarization parameters and the array geometry on the optimal designing strategy.

\section{APPENDIX}

The general case of the CRB, i.e., in the case of $2 \pi \frac{A_{s l}}{\lambda}=L_{s d}$ is given as

$$
\operatorname{CRB}(\boldsymbol{\xi})=\frac{1}{D}\left[\begin{array}{ccc}
F_{\psi, \psi} & -F_{\psi, \omega} & 0 \\
-F_{\omega, \psi} & F_{\omega, \omega} & 0 \\
0 & 0 & \frac{D}{F_{\rho, \rho}}
\end{array}\right]
$$

in which

$$
\begin{aligned}
D & =F_{\omega, \omega} F_{\psi, \psi}-F_{\psi, \omega} F_{\omega, \psi} \\
F_{\omega, \omega} & =\frac{2}{\sigma_{n}^{2}}\|\boldsymbol{s}\|_{2}^{2}\left\|\boldsymbol{d}_{N}\right\|_{2}^{2}\left(4 \pi^{2} \frac{A_{s l}^{2}}{\lambda^{2}} \cos ^{2}(\rho)+L_{s d}^{2} \sin ^{2}(\rho)\right) \\
F_{\rho, \rho} & =\frac{2 N}{\sigma_{n}^{2}}\|\boldsymbol{s}\|_{2}^{2} \|\left(4 \pi^{2} \frac{A_{s l}^{2}}{\lambda^{2}} \sin ^{2}(\rho)+L_{s d}^{2} \cos ^{2}(\rho)\right) \\
F_{\psi, \psi} & =\frac{2 N}{\sigma_{n}^{2}}\|\boldsymbol{s}\|_{2}^{2} \| L_{s d}^{2} \sin ^{2}(\rho) \\
F_{\psi, \omega} & =F_{\omega, \psi}=\frac{2}{\sigma_{n}^{2}}\|\boldsymbol{s}\|_{2}^{2}\left\|\boldsymbol{d}_{N}\right\|_{1}^{2} L_{s d}^{2} \sin ^{2}(\rho)
\end{aligned}
$$

\section{REFERENCES}

[1] T. Bao, A. Breloy, M. N. El Korso, K. Abed-Meraim and H. Ouslimani , "Performance analysis of direction-of-arrival and polarization estimation using a non-uniform linear cold array," in In 2017 Seminar on Detection Systems Architectures and Technologies (DAT), IEEE, vol. 1, 2017, pp. 1-5. https://doi.org/10.1109/DAT.2017.7889165

[2] M. Haardt, M. Pesavento, F. Roemer, and M. N. E. Korso, "Subspace methods and exploitation of special array structures," Electronic Reference in Signal Processing: Array and Statistical Signal Processing (M. Viberg, ed.), vol. 3, pp. 651-717, Academic Press Library in Signal Processing, Elsevier Ltd., Chapter 2.15, ISBN 978-0-12-411597-2, 2014.
[3] H. L. VanTrees, Detection, Estimation and Modulation Theory. New York: Wiley, 1968, vol. 1.

[4] H. Krim and M. Viberg, "Two decades of array signal processing research: the parametric approach," IEEE Signal Processing Mag., vol. 13, no. 4, pp. 67-94, 1996. https://doi.org/10.1109/79.526899

[5] J. Li, P. Stoica, and D. Zheng, "Efficient direction and polarization estimation with a cold array," IEEE Trans. Antennas Propagat., vol. 44, no. 4, pp. 539-547, Apr. 1996. https://doi.org/10.1109/8.489306

[6] J. Li and R. Compton, "Angle and polarization estimation using esprit with a polarizationsensitive array," IEEE Trans. Antennas Propagat., vol. 39, no. 9, pp. 1376-1383, Sep. 1991. https://doi.org/10.1109/8.99047

[7] T. Bao, M. N. El Korso and H. Ouslimani, "Cramer-Rao bound and statistical resolution limit investigation for near-field source localization," in Digital Signal Processing, Elsevier, vol. 48, No. 1, 2016, p. 137-147. https://doi.org/10.1016/j.dsp.2015.09.019

[8] R. Boyer and S. Miron, "Study of the asymptotic cramer-rao bound for the cold uniform linear array," in Physical Communication, Elsevier, 1982. https://doi.org/10.1016/j.phycom.2012.02.004

[9] R. Boyer, "Analysis of the COLD uniform linear array," in Proc. IEEE Int. Work. Signal Processing, Wireless Communications, Perugia, Italy, 2009. https://doi.org/10.1109/SPAWC.2009.5161848

[10] J. Tabrikian, R. Shavit, and D. Rahamim, "An efficient vector sensor configuration for source localization," IEEE Signal Process. Letters, vol. 11, pp. 690-693, Aug. 2004. https://doi.org/10.1109/LSP.2004.831682

[11] M. N. El Korso, R. Boyer, A. Renaux, and S. Marcos, "Statistical resolution limit of the uniform linear cocentered orthogonal loop and dipole array," IEEE Trans. Signal Processing, vol. 59, no. 1, pp. 425-431, Jan. 2011. https://doi.org/10.1109/TSP.2010.2083657

[12] E. Chaumette, J. Galy, A. Quinlan, and P. Larzabal, "A new Barankin bound approximation for the prediction of the threshold region performance of maximum likelihood estimators," IEEE Trans. Signal Processing, vol. 56, no. 11, pp. 5319-5333, Nov. 2008. https://doi.org/10.1109/TSP.2008.927805

[13] E. Chaumette, A. Renaux, and M. N. El Korso, "A class of weiss-weinstein bounds and its relationship with the bobrovskymayer-wolf-zakai bounds," in IEEE Transactions on Information Theory, vol. 63, No. 4, 2017, pp. 2226-2240. https://doi.org/10.1109/TIT.2017.2671883

[14] P. Forster and P. Larzabal, "On lower bounds for deterministic parameter estimation," in Proc. of IEEE Int. Conf. Acoust., Speech, Signal Processing, Orlando, Fl, 2002. https://doi.org/10.1109/ICASSP.2002.5744000 
[15] K. Todros and J. Tabrikian, "General classes of performance lower bounds for parameter estimation Part I: Nonbayesian bounds for unbiased estimators," IEEE Trans. Inform. Theory, vol. 56, pp. 5045-5063, Oct. 2010. https://doi.org/10.1109/TIT.2010.2059850

[16] M. N. E. Korso, A. Renaux, R. Boyer, and S. Marcos, "Deterministic performance bounds on the mean square error for near field source localization," IEEE Trans. Signal Process., vol. 61, pp. 871-877, Apr. 2013. https://doi.org/10.1109/TSP.2012.2229990

[17] S. T. Smith, "Statistical resolution limits and the complexified Cramér Rao bound," IEEE Trans. Signal Processing, vol. 53, pp. 1597-1609, May 2005. https://doi.org/10.1109/TSP.2005.845426

[18] J.-P. Delmas, M. N. El Korso, H. Gazzah and M. Castella, "Crb analysis of planar antenna array for optimizing near-field source localization," in Signal Processing Journal, Elsevier, vol. 127, No. 1, 2016, p. 117-134. https://doi.org/10.1016/j.sigpro.2016.02.021

[19] M. N. El Korso, R. Boyer, A. Renaux, and S. Marcos, "Statistical resolution limit for the multidimensional harmonic retrieval model: Hypothesis test and Cramér-Rao bound approaches," EURASIP Journal on Advances in Signal Processing, vol. special issue on Advances in Angle-of-Arrival and Multidimensional Signal Processing for Localization and Communications, pp. 1-14, Jul. 2011. https://doi.org/10.1186/1687-6180-2011-12

[20] H. Cramér, Mathematical Methods of Statistics. New York: Princeton University, Press, 1946.

[21] S. M. Kay, Fundamentals of Statistical Signal Processing : Estimation Theory. NJ: Prentice Hall, 1993, vol. 1.

[22] A. Mennad, S. Fortunati, M. N. El Korso, A. Younsi, A. M. Zoubir and A. Renaux, "Slepian-bangs-type formulas and the related misspecified cramér-rao bounds for complex elliptically symmetric distributions," in Elsevier Signal Processing Journal, vol. 142, 2018, pp. 320-329. https://doi.org/10.1016/j.sigpro.2017.07.029

[23] S. Kullback and R. A. Leibler, "On information and sufficiency," in Ann. Math. Stat., vol. 22, No. 1, 1951, pp. 79-86. https://doi.org/10.1214/aoms/1177729694

[24] A. Graham, "Kronecker products and matrix calculus with applications," in Wiley, New York, 1982.

[25] G. H. Golub and C. F. V. Loan, Matrix Computations. London: Johns Hopkins, 1989.

[26] K. Bell, Y. Ephraim, and H. L. V. Trees, "Ziv Zakai lower bounds in bearing estimation," in Proc. of IEEE Int. Conf. Acoust., Speech, Signal Processing, vol. 5, Detroit, Michigan, 1995, pp. 2852-2855. https://doi.org/10.1109/ICASSP.1995.479888

Tao Bao was born in Bayannur, Inner Mongolia Autonomous Region, China, in 1983. She received the B.S., M.S. and Ph.D. degrees in information and communication engineering from Northwestern Polytechnical University, China, in 2005, 2008, and 2010, respectively. From 2010, she has been with the provincial electronics experiment teaching demonstration center, Northwestern Polytechnical University, where she is currently a deputy director. From 2013 to 2014, she was a visiting scholar at the Paris Ouest Nanterre La Defense University. Her major research interests include statistical signal processing, array signal processing and space-time adaptive processing in wireless communications.

Mohammed Nabil El Korso was born in Oran, Algeria. He received the M.Sc. in Electrical Engineering from the National Polytechnic School, Algeria in 2007. He obtained the Master Research degree in Signal and Image Processing from Paris-Sud XI University/Supelec, France in 2008. In, 2011, he obtained his
Ph.D. degree from Paris-Sud XI University. Between 2011 and 2012, he was a research scientist in the Communication Systems Group at Technische Universitat Darmstadt, Germany. He was a temporary assistant professor at l'école normale supérieure de Cachan, between 2012 and 2013. Currently, he is assistant professor at University of Paris Ouest Nanterre la Défense and a member of LEME (EA4416) laboratory. His research interests include statistical signal processing, estimation/detection theory with applications to array signal processing. 